\title{
Alteration of immune profiles is associated with pulmonary function and symptoms in patients with chronic obstructive pulmonary disease
}

\author{
SIXIANG LI ${ }^{1 *}$, SHUANG ZHAO $^{1 *}$, ZHENRU WU $^{2}$, FANGFANG WANG $^{3}$ and WEIMIN LI ${ }^{1}$ \\ ${ }^{1}$ Department of Respiratory and Critical Care Medicine, ${ }^{2}$ Pathology Research Laboratory and \\ ${ }^{3}$ Hematology Research Laboratory, West China Hospital, Sichuan University, Chengdu, Sichuan 610041, P.R. China
}

Received October 30, 2019; Accepted June 10, 2021

DOI: $10.3892 / \mathrm{mmr} .2021 .12382$

\begin{abstract}
Inflammation serves a key role in chronic obstructive pulmonary disease (COPD). However, changes in the immune profiles of patients with COPD remain unclear. The present prospective observational study aimed to determine the expression profiles of immune cells and inflammatory factors of patients with COPD and healthy controls, and to analyze the relationship between immune profiles and smoking history. A total of 140 subjects were enrolled in the present study between September 2018 and April 2019 at West China Hospital of Sichuan University (Chengdu, China). These included 87 patients with stable COPD and 24 patients with acute exacerbated COPD, as well as 29 healthy controls. Baseline characteristics were recorded during the screening period, and levels of immune cells were examined using flow cytometry. In addition, levels of inflammatory factors were measured using ELISAs. The results revealed increased expression of the $\mathrm{CD} 64^{+} / \mathrm{CD} 14^{+}$mononuclear phagocyte system (MPS) and $\mathrm{CD}_{16}{ }^{+} \mathrm{CD}_{66}{ }^{+}$neutrophils, and decreased expression of $\mathrm{CD}^{+} \mathrm{CD}^{+} \mathrm{T}$ cells and $\mathrm{CD}^{+} \mathrm{CD}^{+} \mathrm{T}$ cells (all $\mathrm{P}<0.05$ ) in the peripheral blood of patients with COPD and smokers relative to non-smoking controls. In addition, significant differences were observed in protein levels of IL- 6 , IL-1 $\beta$, TNF- $\alpha$, TGF- $\alpha$, IFN- $\gamma$, IL-8, IL-17A and CRP among the three groups (all $\mathrm{P}<0.05)$. Furthermore, the IL-17A, TNF and NF- $\mathrm{B}$ signaling pathways were found to serve a key role in the inflammatory network of COPD. Pearson's correlation analysis revealed a positive correlation between $\mathrm{CD}^{+} \mathrm{T}$ lymphocyte counts and
\end{abstract}

Correspondence to: Professor Weimin Li, Department of Respiratory and Critical Care Medicine, West China Hospital, Sichuan University, 17 3rd Part of Renmin Road, Chengdu, Sichuan 610041, P.R. China

E-mail: weimi003@scu.edu.cn

*Contributed equally

Key words: immune cell, inflammatory factor, chronic obstructive pulmonary disease, smoking pulmonary function, and a negative correlation with smoking and exacerbations. Furthermore, neutrophils and MPS were negatively associated with pulmonary function, and IL-8 was positively associated with cough. There was also a negative association between CRP and IL-17A with pulmonary function but a positive correlation with symptoms and exacerbation. Club cell secretory protein was also negatively associated with emphysema parameters. In conclusion, the present findings revealed significant differences in profiles of immune factors among patients with COPD, smokers and non-smoking controls and their association with clinical characteristics. The clinical trial registration number of the present study is: ChiCTR1800015700 (registered with http://www.chictr.org. cn/index.aspx, 2018/04/16).

\section{Introduction}

Chronic obstructive pulmonary disease (COPD) ranks fourth among disease-related causes of death worldwide (1). COPD, regarded as a chronic systemic inflammatory disease, is characterized by persistent limitation of airflow due to airway and/or alveolar abnormalities (2). Cigarette smoking (CS) is the main cause of COPD, although other factors have also been identified (3). Furthermore, its pathogenesis is based on genetic determinants, lung growth, as well as environmental stimuli, such as oxidative stress, inflammation, extracellular matrix proteolysis, and apoptotic and autophagic cell death (4). In addition, bacterial and/or viral infections have been reported to exacerbate COPD (3). Airway inflammation is a key driver of COPD progression, resulting in several phenotypes with different patterns (5). Attempts have been made to segregate patients with COPD into different subtypes based on immune profiles, such as neutrophil- or eosinophil-associated COPD (6), with evidence indicating corticosteroids to be the most effective in patients with COPD with eosinophilic inflammation $(5,7)$. In addition, novel biological therapies targeting specific inflammatory biomarkers have been explored. For instance, anti-IL5 therapy in COPD reduces exacerbations in patients with a high blood eosinophil count by $20 \%$ (8).

Recently, potential biomarkers such as IL-5 and TNF- $\alpha$ have been considered effective in targeted anti-inflammatory therapy (9). Multiscale models of CS-induced COPD have 
enabled identification of several positive feedback loops, such as M1 and M2 transformation and balance, as well as network elements, and these have served a determinant role in CS-induced immune response and COPD progression (10,11). An increased proportion of infiltrating macrophages, as well as a reduction of $\mathrm{CD}^{+}$and $\mathrm{CD} 8^{+} \mathrm{T}$ cells, are both independently associated with smoking status or the level of airflow limitation $(10,11)$. CRP is associated with elevation of acute exacerbated COPD (AECOPD), while CRP-guided prescription of antibiotics for AECOPD results in less use of antibiotics with no evidence of harm (12). Levels of IL-6 are associated with the degree of airflow limitation (13), and both IL-6 and IL-8 are associated with emphysema severity (14). Similarly, IL-1 $\beta$, IL-17A, TNF- $\alpha$ and IFN- $\gamma$ have also been linked to persistent airway inflammation and greater exacerbation (15-17). In addition, pulmonary surfactant proteins might predict relapse of patients with AECOPD (18), while lack of club cell secretory protein $(\mathrm{CC} 16)$ in the lungs results in enhanced airway remodeling in COPD (19). Members of the extracellular MMP family of proteins are associated with emphysema pathogenesis (20). Consequently, MMP inhibitors have been developed as novel therapeutics, with doxycycline approved by the Food and Drug Administration (20). It is possible that more biomarkers could promote the understanding of COPD pathogenesis and development of targeted therapies. However, the complexity of these biomarkers poses challenges in distinguishing between COPD phenotypes and endotypes. It is, therefore, imperative to focus on biomarkers that have a clear distinction of specific clinical phenotypes or endotypes (21).

The present study analyzed the peripheral blood expression of immune cells and serum levels of 20 inflammatory factors with the aim to elucidate the complex network of immune profiles in COPD and airway inflammatory patterns. The present findings may provide insight to guide the future development of novel therapies.

\section{Materials and methods}

Study patients and ethics. A total of 140 subjects with mean age of 65.13 were enrolled in the study between September 2018 and June 2019. In total, 87 patients with stable COPD (62 male, 25 female, age: Mean \pm SEM 64.94 \pm 0.97 ) were recruited at the Outpatient Department of West China Hospital, Sichuan University (Chengdu, China). Inclusion criteria used for the recruitment included: i) Diagnosed with COPD by respiratory physician; ii) performed pulmonary function test [forced expiratory volume in $1 \mathrm{sec}$ (FEV1)/forced vital capacity (FVC) $\leq 70 \%$ ] and chest CT scan; iii) no malignant tumor or autoimmune disease; iv) no liver and kidney failure or special infections such as HIV; v) no exacerbation in 3 months; vi) no other chronic and acute severe respiratory diseases; and vii) no chronic or acute systemic infections. A total of 24 patients with AECOPD (17 male, 7 female, age: Mean \pm SEM 67.00 \pm 2.13 ) admitted at West China Hospital were enrolled using the following inclusion criteria: i) Main diagnosis for admission was AECOPD; ii) pulmonary function test (FEV1/FVC $\leq 70 \%)$ and chest CT scan were performed; iii) no malignant tumor or autoimmune diseases; iv) no liver and kidney failure or special infections such as HIV; and v) no other severe chronic or acute respiratory disease. For those patients for whom pulmonary function test and chest CT scan were not performed, medical reports for the past year were required. COPD was defined according to international guidelines (2), while severity of airflow obstruction was graded using current Global Initiative for Chronic Obstructive Lung Disease criteria (2). A total of 29 healthy controls (18 non-smokers and 11 smokers, 18 male, 11 female, age: Mean \pm SEM 64.15 \pm 1.34 ) were recruited at the Physical Examination Center of West China Hospital (Chengdu, China) using the following inclusion criteria: i) Pulmonary function test (FEV1/FVC $<70 \%)$ and chest CT scan were performed, indicating normal pulmonary function; ii) no malignant tumor or autoimmune disease; iii) no heart, liver and kidney failure or special infections; iv) no other chronic or acute respiratory disease; and v) no chronic or acute systemic infections. Spirometry parameters were measured according to the recommendations of the European Respiratory Society, and then expressed as a percentage of the predicted (22). The modified Medical Research Council dyspnea scale (mMRC) was adopted as the classification criterion for symptom assessment (23), detailed description of baseline characteristics was recorded by the respiratory physician. According to smoking history, all the subjects were divided into four groups: Smoking (73) and nonsmoking (24) COPD patients and smoking (8) and nonsmoking (17) controls. The present study was approved by The Ethics Committee of West China Hospital, Sichuan University (Chengdu, China) in accordance with the Declaration of Helsinki (24). All patients provided written informed consent before participating in the study.

Flow cytometry. Fresh peripheral blood $(2 \mathrm{ml})$ anticoagulated in EDTA was collected from each patient and processed within $24 \mathrm{~h}$. Peripheral blood was divided into 2 tubes $(1 \mathrm{ml})$. The corresponding antibody $(2 \mu \mathrm{l})$ was added to each tube (tube 1: CD45,PE-cy5.5;CD14, FITC;CD64, PE;CD66, APC;CD16, PE-cy7; tube 2: CD45,PE-cy5.5;CD3,PE; CD8, APC-cy7;CD4, FITC;CD56, PE-cy7;CD19, APC) and incubated for $30 \mathrm{~min}$ in the dark at room temperature, then mixed with red blood cell lysis solution (FCM Lysing solution for BC, Hangzhou Multi Sciences (Lianke) Biotech Co., Ltd.) with the ratio of 1:3, lysing for $\sim 10$ min until the solution was clear. Following centrifugation $\left(500 \mathrm{x} \mathrm{g}\right.$ for $5 \mathrm{~min}$ at $4^{\circ} \mathrm{C}$ ), the supernatant was removed and washed by $500 \mu \mathrm{l}-1 \mathrm{ml}$ PBS for three times, centrifuged at $400 \mathrm{x} \mathrm{g}$ for $5 \mathrm{~min}$ at $4^{\circ} \mathrm{C}$, and then resuspended in $200 \mu \mathrm{l}$ PBS. The cells were stored on ice in the dark, or in a refrigerator at $4^{\circ} \mathrm{C}$ until use. Samples were analyzed using multicolor flow cytometry (Navios EX flow cytometer; Beckman Coulter, Inc.) based on $\mathrm{CD} 45^{+}$human leukocytes. $\mathrm{CD} 64^{+} / \mathrm{CD} 14^{+}$represented the monocyte-macrophage system (MPS), $\mathrm{CD}^{+} \mathrm{T}$ lymphocytes were divided into $\mathrm{CD} 4^{+}$and $\mathrm{CD} 8^{+}$subsets, while $\mathrm{CD}^{+}{ }^{+} \mathrm{CD} 4{ }^{-} \mathrm{CD} 8{ }^{-}$was selected as the marker for $\gamma \delta \mathrm{T}$ cells. In addition, $\mathrm{CD}^{+} \mathrm{CD}^{2} 6^{+}$marked natural killer $\mathrm{T}$ lymphocytes (NKTs), while $\mathrm{CD} 3{ }^{-} \mathrm{CD} 56^{+}$marked natural killer (NK) cells. Antibodies ready to use were acquired from eBioscience (Thermo Fisher Scientific, Inc.; CD14 Monoclonal Antibody, FITC, human, cat. no. 11-0149-42; CD64 Monoclonal Antibody, PE, human, cat. no. 12-0649-42; CD66 Monoclonal Antibody, APC, human, cat. no. 17-0668-42; CD16 Monoclonal Antibody, PE-cy7, human, cat. no. 25-0168-42; 
CD3 Monoclonal Antibody, PE, human, cat. no. 12-0038-42; CD8 Monoclonal Antibody, APC-cy7, human, cat. no. A15448; CD4 Monoclonal Antibody, FITC, human, cat. no. 11-0049-42; CD56 Monoclonal Antibody, PE-cy7, human, cat. no. 25-0567-42; CD19 Monoclonal Antibody, APC, human, cat. no. 17-0199-42; CD45 Monoclonal Antibody, PE-cy 5.5, human, cat. no. 35-0459-42), and cells were stained according to the manufacturer's recommendations. All the antigens were human source and stored at $4^{\circ} \mathrm{C}$ in the dark. Monocyte-macrophage-granulocyte included antigens of CD45, CD14, CD64, CD66 and CD16, while lymphocyte-NK-NK included CD45, CD3, CD8, CD4, CD56 and CD19. FlowJo version 10.0.7 software (FlowJo LLC) was used for analysis.

Analysis of inflammatory factors. A total of $5 \mathrm{ml}$ peripheral EDTA-anticoagulated blood was collected from each patient, $2 \mathrm{ml}$ used for FCM, the rest $(3 \mathrm{ml})$ centrifuged at $1,600 \mathrm{x}$ for $15 \mathrm{~min}$ at $4^{\circ} \mathrm{C}$. The resulting plasma was stored at $-79^{\circ} \mathrm{C}$ for subsequent ELISA tests and multi-plex panel tests.

ELISA. The levels of five biomarkers were measured using ELISAs. The plasma stored at $-79^{\circ} \mathrm{C}$ was defrosted in a constant temperature water bath to $37^{\circ} \mathrm{C}$. CRP (Human C-Reactive Protein/CRP Immunoassay kit; cat. no. DCRP00), CC16 (Human Uteroglobin Quantikine ELISA kit; cat. no. DUGB00) and TGF- $\beta$ (Human TGF-beta Quantikine ELISA kit; cat. no. DB100B) levels were assessed using Quantikine ELISA kits (all R\&D Systems, Inc.), while fibrinogen(Human Fibrinogen ELISA kit; ab208036) and neutrophil elastase (Human PMN Elastase ELISA kit; ab119553) were analyzed using Simple Step ELISA kits (Abcam) according to the to the instructions of the respective manufacturer's instructions.

Multi-plex panel. Two multi-factor panels, including 15 biomarkers, were tested using the Multiplexed kit (Magnetic Luminex ${ }^{\circledR}$ Assay, Human Premixed Multi-Analyte kit; LXSAHM, R\&D Systems, Inc.) based on the Luminex 200 system with XPONENT 3.1 (Luminex Corporation). One panel tested the levels of 13 analytes, including IL-6, TNF- $\alpha$, IL-1 $\beta$, IFN- $\gamma$, IL-8, IL-33, IL-17A, IL-4, IL-5, IL-13, TGF- $\alpha$, Human growth-regulated oncogene $\alpha(\mathrm{GRO}-\alpha)$ and pulmonary surfactant-associated protein D (SP-D), while the other was used to assess myeloperoxidase (MPO) and MMP-9 (Supplementary Materials).

Bioinformatics. Proteins (IL-6, IL-1 $\beta$, TNF- $\alpha$, IFN- $\gamma$, IL-8, IL-33, IL-17A, TGF- $\alpha$, GRO- $\alpha$, CRP) and their functional interactions were analyzed using the Search Tool for the Retrieval of Interacting Genes/Proteins (STRING) database version 11.0 (25), and enriched Kyoto Encyclopedia of Genes and Genomes pathways were determined using STRING [10 items (human)-STRING interaction network (string-db.org)].

Statistical analysis. All samples were tested 3 times. Continuous variables are presented as the mean \pm standard error of the mean and were analyzed using SPSS 22.0 (IBM Corp.). A normality test was performed using a P-P diagram in SPSS 22.0. One-way ANOVA and Tukey's post hoc test were used for comparisons of normally distributed continuous variables among different groups. Kruskal Wallis and Dunn's post hoc test were used for comparisons of non-normally distributed continuous variables among different groups. In addition, $\chi^{2}$ and Bonferroni's correction were used for categorical variables. Pearson's correlation was used to assess the relationship among continuous variables, while Spearman's correlation test was used for categorical data. Figures were generated using FlowJo version 10.0.7 software (FlowJo LLC) and GraphPad Prism 8 (GraphPad Software, Inc.). $\mathrm{P}<0.05$ was considered to indicate a statistically significant difference.

\section{Results}

Clinical characteristics of the patients. A total of 87 patients with stable COPD and 24 patients with AECOPD, as well as 29 healthy controls were recruited in the study. There were no significant differences in terms of age, sex and BMI among the three groups (Table I). As expected, the proportion of smokers was higher among patients with COPD compared with the controls (COPD vs. AECOPD vs. controls; 75.9 vs. 62.5 vs. $37.9 \%$, respectively), and the lung function (FEV1/FVC, FEV1\% and maximal mid expiratory flow (MMEF)75/25*) of patients with COPD was worse compared with controls (Table I). AECOPD had more group D patients with severe airflow limitation (stage III, 38.89 vs. $22.50 \%$; stage IV, 50.00 vs. $28.75 \%$ ) compared with COPD (Table I). The score of mMRC assessment was higher in patients with AECOPD compared with patients with stable COPD (3.61 vs. 2.24). The most common drug therapy for patients with COPD and AECOPD was long-acting muscarinic antagonist (LAMA) + long-acting beta-2 agonist (LABA) + inhaled corticosteroid (ICS; 63.29 and $45 \%$, respectively), while $17.86 \%$ of patients with stable COPD did not receive drug therapy. In conclusion, patients with COPD who smoked had worse lung function compared with healthy controls, patients with AECOPD were severe and needed more medication than stable patients with COPD.

Immune cells expression profiles. The flow cytometry results and workflow are shown in Figs. 1 and 2. Following staining, living cells were imaged and the $\mathrm{CD} 45^{+}$subset were gated as human leukocyte There were significant differences in $\mathrm{CD}^{+}$ $\mathrm{T}$ lymphocytes $(\mathrm{P}<0.001)$, including the $\mathrm{CD}^{+} \mathrm{T}(\mathrm{P}<0.001)$ and $\mathrm{CD}^{+} \mathrm{T}(\mathrm{P}=0.004)$ subsets, $\mathrm{CD} 14^{+}(\mathrm{P}=0.027)$ and $\mathrm{CD} 64^{+}$ $(\mathrm{P}=0.026) \mathrm{MON}$ and $\mathrm{CD} 16^{+} \mathrm{CD} 6^{+}$neutrophil $(\mathrm{P}=0.011)$ proportions across the study groups (Table II; Fig. 3). $\mathrm{CD}^{+} 6^{+} \mathrm{CD} 6^{+}$neutrophils and MPS (CD14 ${ }^{+}$and $\mathrm{CD} 64^{+}$subsets) were highly expressed, while $\mathrm{CD} 4^{+}$and $\mathrm{CD} 8^{+} \mathrm{T}$ lymphocytes were expressed at lower levels in patients with stable COPD and patients with AECOPD compared with healthy controls (Table II). CD4 ${ }^{+} \mathrm{T}$ lymphocyte counts were decreased in patients with stable COPD compared with controls, and in AECOPD compared with stable COPD (AECOPD vs. COPD vs. controls, 6.50 vs. 9.04 vs. $12.36 \%$, respectively). $\mathrm{CD}^{+} 4^{+}$and $\mathrm{CD} 14^{+} \mathrm{MON}$ tended to be increased in AECOPD compared with stable COPD and controls (CD64+ ${ }^{+} \mathrm{MON}$ : AECOPD vs. COPD vs. controls, 4.53 vs. 3.94 vs. $2.85 \%$; $\mathrm{CD}_{14}{ }^{+} \mathrm{MON}$ : AECOPD vs. COPD vs. controls, 4.57 vs. 4.19 vs. $3.04 \%$, respectively). $\mathrm{CD} 14^{+} \mathrm{CD} 64^{+} \mathrm{MON}$ levels were increased in AECOPD compared with controls (3.82 vs. 2.53\%). Furthermore, $\mathrm{CD} 19^{+} \mathrm{B}$ lymphocytes, $\mathrm{CD}^{+} \mathrm{CD}^{+} 6^{+} \mathrm{NKT}$ and 
Table I. Clinical baseline characteristics of patients with COPD, patients with AECOPD and healthy controls.

\begin{tabular}{|c|c|c|c|c|}
\hline Baseline features & $\operatorname{COPD}(\mathrm{n}=87)$ & $\operatorname{AECOPD}(n=24)$ & Healthy controls $(n=29)$ & P-value \\
\hline Age, years (mean \pm SEM) & $64.94 \pm 0.97$ & $67.00 \pm 2.13$ & $64.15 \pm 1.34$ & 0.519 \\
\hline Male, n $(\%)$ & $62(76.7)$ & $17(75.0)$ & $18(62.7)$ & 0.444 \\
\hline $\mathrm{BMI}($ mean $\pm \mathrm{SEM})$ & $22.05 \pm 0.58$ & $19.70 \pm 1.75$ & $21.23 \pm 1.48$ & 0.299 \\
\hline Smoking history, n (\%) & $66(75.9)$ & $15(62.5)$ & $11(37.9)$ & $0.001^{\mathrm{a}}$ \\
\hline Smoking amount, pack-year (mean \pm SEM) & $31.59 \pm 3.26$ & $22.38 \pm 4.23$ & $9.71 \pm 3.57$ & $0.001^{\mathrm{a}}$ \\
\hline Exacerbation, $\mathrm{n}(\%)$ & $51(58.62)$ & $24(100)$ & & $0.001^{\mathrm{a}}$ \\
\hline \multicolumn{5}{|l|}{ Pulmonary function } \\
\hline No. of patients analyzed (n) & 78 & 16 & 22 & \\
\hline FEV1/FVC (mean \pm SEM) & $50.94 \pm 1.24$ & $46.74 \pm 2.32$ & $78.91 \pm 1.37$ & $0.001^{\mathrm{a}}$ \\
\hline FEV1\% $($ mean \pm SEM $)$ & $51.83 \pm 2.44$ & $40.22 \pm 4.47$ & $96.23 \pm 2.27$ & $0.001^{\mathrm{a}}$ \\
\hline RV/TLC (mean \pm SEM) & $142.14 \pm 8.61$ & $145.06 \pm 14.72$ & $110.58 \pm 4.34$ & 0.281 \\
\hline MMEF75/25 (mean \pm SEM) & $22.45 \pm 1.78$ & $13.49 \pm 1.87$ & $71.52 \pm 3.42$ & $0.001^{\mathrm{a}}$ \\
\hline \multicolumn{5}{|l|}{ GOLD stage $^{\mathrm{b}}$} \\
\hline No. of patients analyzed & 84 & 22 & & \\
\hline $\mathrm{A}, \mathrm{n}(\%)$ & $23(27.38)$ & $0(0.00)$ & & $0.001^{\mathrm{a}}$ \\
\hline $\mathrm{B}, \mathrm{n}(\%)$ & $22(26.19)$ & $3(13.64)$ & & $0.001^{\mathrm{a}}$ \\
\hline $\mathrm{C}, \mathrm{n}(\%)$ & $8(9.52)$ & $1(4.54)$ & & $0.001^{\mathrm{a}}$ \\
\hline $\mathrm{D}, \mathrm{n}(\%)$ & $31(36.9)$ & $18(81.82)$ & & $0.001^{\mathrm{a}}$ \\
\hline \multicolumn{5}{|l|}{ Stage of Airflow limitation ${ }^{b}$} \\
\hline No. of patients analyzed & 80 & 18 & & \\
\hline $\mathrm{I}($ mild $), \mathrm{n}(\%)$ & $12(15)$ & $1(5.56)$ & & $0.001^{\mathrm{a}}$ \\
\hline II (moderate), n (\%) & $27(33.75)$ & $1(5.26)$ & & $0.001^{\mathrm{a}}$ \\
\hline III (severe), n (\%) & $18(22.5)$ & $7(38.89)$ & & $0.001^{\mathrm{a}}$ \\
\hline IV (very severe), n (\%) & $23(28.75)$ & $9(50)$ & & $0.001^{\mathrm{a}}$ \\
\hline \multicolumn{5}{|l|}{ Assessments } \\
\hline No. of patients analyzed & 84 & 20 & & \\
\hline mMRC (mean \pm SEM) & $2.24 \pm 0.11$ & $3.61 \pm 0.14$ & & $0.001^{\mathrm{a}}$ \\
\hline Cough $($ mean \pm SEM) & $1.88 \pm 0.14$ & $2.09 \pm 0.45$ & & 0.528 \\
\hline Sputum (mean \pm SEM) & $5.22 \pm 0.33$ & $5.64 \pm 0.28$ & & 0.724 \\
\hline \multicolumn{5}{|l|}{ Drug therapy } \\
\hline No. of patients analyzed & 84 & 20 & & \\
\hline 0 (none), n $(\%)$ & $15(17.86)$ & $0(0)$ & & \\
\hline 1 (LAMA/LABA), n (\%) & $8(9.52)$ & $0(0)$ & & \\
\hline $2(\mathrm{LABA}+\mathrm{ICS}), \mathrm{n}(\%)$ & $13(15.48)$ & $3(15)$ & & \\
\hline 3 (LAMA+LABA+ICS), n (\%) & $53(63.29)$ & $9(45)$ & & \\
\hline $4(\mathrm{LAMA}+\mathrm{LABA}), \mathrm{n}(\%)$ & $3(3.57)$ & $8(40)$ & & \\
\hline
\end{tabular}

${ }^{\mathrm{a}} \mathrm{P}<0.001 .{ }^{\mathrm{b}} \mathrm{GOLD}$ stage and airflow limitation severity in accordance with GOLD 2018 report, severity of airflow limitation: FEV1\%, $\geq 80$, stage I; FEV1\%, 50-79, stage II; FEV1\%, 30-49, stage III; and FEV1\%, <30, stage IV. COPD, chronic obstructive pulmonary disorder; AE, acute exacerbated; FEV1, forced expiratory volume in $1 \mathrm{sec}$; FVC, forced vital capacity; RV, residual volume; TLC, total lung compacity; MMEF75/25, maximal mid expiratory flow; GOLD, Global Initiative for Chronic Obstructive Lung Disease; mMRC, modified Medical Research Council dyspnea scale; LAMA, long-acting muscarinic antagonist; LABA, long-acting beta-2 agonist; ICS, inhaled corticosteroid. Some patients were unable to finish complete lung function test, so patients able to complete the test were analyzed.

CD3-CD56 ${ }^{+} \mathrm{NKs}$ levels were not significantly different among the three groups.

There were 73 smoking patients with COPD and 24 non-smoking patients with COPD, as well as 8 smoking controls and 17 non-smoking controls. The majority of smokers were male, and their age was comparable among the four groups. There were also significant differences in $\mathrm{CD} 3{ }^{+} \mathrm{CD} 8^{+} \mathrm{T}$ lymphocyte
$(\mathrm{P}<0.001), \mathrm{CD}_{4}{ }^{+}(\mathrm{P}=0.014)$ and $\mathrm{CD}^{+} 4^{+}(\mathrm{P}=0.020) \mathrm{MON}$, and $\mathrm{CD}^{+} 6^{+} \mathrm{CD}_{6}{ }^{+}$neutrophil $(\mathrm{P}=0.002)$ proportions only between smokers and non-smokers (Table III). CD4 ${ }^{+} \mathrm{T}$ lymphocytes levels were sharply decreased in patients with COPD compared with non-smoking controls (smoking COPD vs. non-smoking COPD vs. non-smoking controls: 8.16 vs. 9.42 vs. $12.75 \%$ ). Levels of $\mathrm{CD}^{+} \mathrm{T}$ lymphocytes were decreased in both patients 

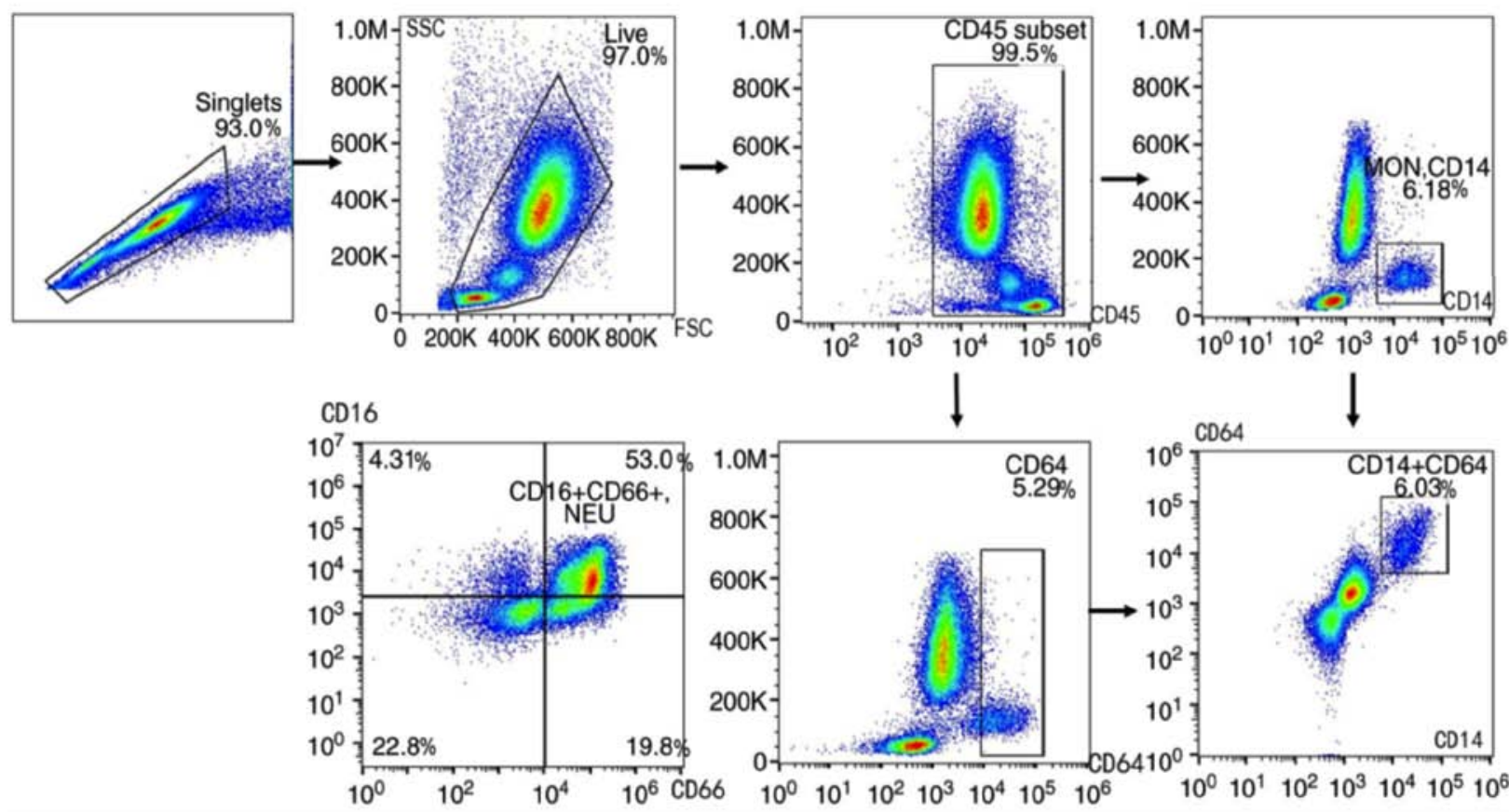

Figure 1. Flowchart of mononuclear phagocyte system antigen staining of flow cytometry. Ratio of $\mathrm{CD}_{14}{ }^{+}, \mathrm{CD} 4^{+}$monocyte and $\mathrm{CD} 16^{+} \mathrm{CD} 66^{+}$neutrophils among $\mathrm{CD}^{+} 5^{+}$leukocytes (\%). FSC, forward scatter; SSC, side scatter; MON, monocyte; NEU, neutrophil.

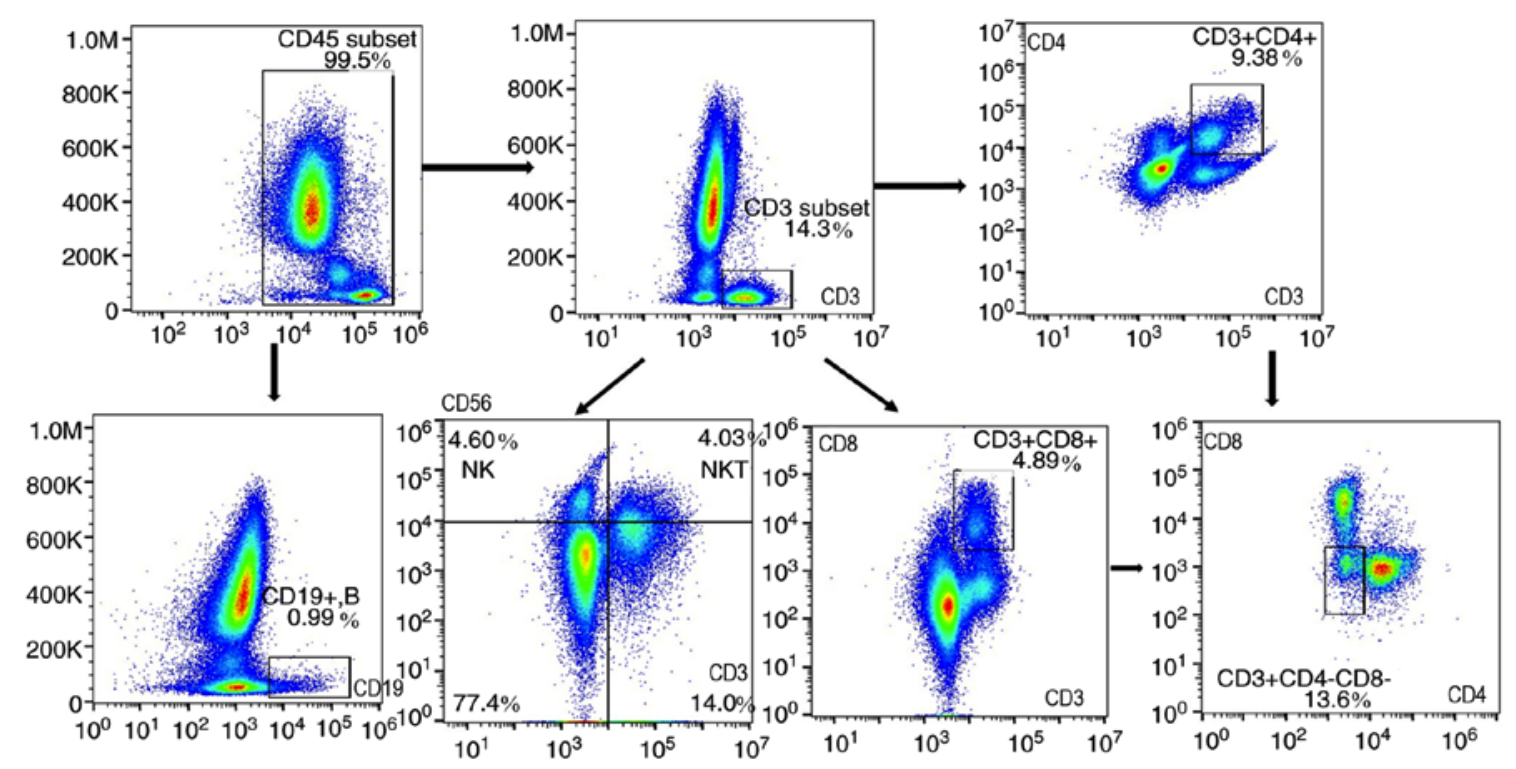

Figure 2. Flowchart of lymphocytes antigen staining of flow cytometry. Ratio of $\mathrm{CD}^{+}{ }^{+} \mathrm{CD} 4^{+} \mathrm{T}$ cells, $\mathrm{CD}^{+} \mathrm{CD} 8^{+} \mathrm{T}$ cells, $\mathrm{CD} 3^{+} \mathrm{CD} 4{ }^{-} \mathrm{CD} 8 \mathrm{~T}$ cells $(\gamma \delta \mathrm{T}$ cells), $\mathrm{CD}^{+} \mathrm{CD} 56^{+} \mathrm{NKT}$ cells, $\mathrm{CD} 3{ }^{-} \mathrm{CD} 56^{+} \mathrm{NK}$ cells and $\mathrm{CD} 19^{+} \mathrm{B}$ cells among $\mathrm{CD} 45^{+}$leukocytes (\%). NKT, natural killer T; NK, natural killer.

with COPD and smoking controls compared with non-smoking controls (smoking COPD vs. non-smoking COPD vs. smoking controls vs. non-smoking controls: 5.15 vs. 5.98 vs. 5.33 vs. 9.11\%). While $\mathrm{CD}_{6}{ }^{+} \mathrm{CD} 16^{+}$neutrophils (smoking COPD vs. non-smoking COPD vs. smoking controls vs. non-smoking controls: 68.83 vs. 64.99 vs. $66.41 \%$ vs. $56.04 \%) \mathrm{MON}\left(\mathrm{CD} 14^{+}\right.$ and $\mathrm{CD} 64^{+}$subsets) were more highly expressed in patients with COPD and smoking controls compared with non-smoking controls (Table III). CD14 ${ }^{+} \mathrm{CD} 64^{+} \mathrm{MON}$ levels were increased in smokers compared with non-smokers (smoking COPD vs. non-smoking COPD, 3.49 vs. $2.86 \%$; smoking control vs. non-smoking control, 3.58 vs. $2.03 \%$ ). Furthermore, the levels of $\mathrm{CD} 19^{+} \mathrm{B}$ lymphocytes, $\mathrm{CD} 3^{+} \mathrm{CD} 56^{+}$NKTs and $\mathrm{CD} 3{ }^{-} \mathrm{CD} 56^{+}$ NKs were not significantly different among the four groups.

Inflammatory biomarkers. Higher IL-6 (COPD vs. AECOPD vs. controls, 6.95 vs. 20.51 vs. 3.82), IL-1 $\beta$ (COPD vs. AECOPD vs. controls, 6.69 vs. 7.24 vs. 4.09 ) and TGF- $\alpha$ (COPD vs. AECOPD vs. controls, 2.15 vs. 2.20 vs. 1.46) levels were observed in the COPD and AECOPD groups compared with healthy controls. The levels of TNF- $\alpha$ (AECOPD vs. COPD vs. controls, 21.71 vs. 11.23 vs. 8.56 ), IFN- $\gamma$ (AECOPD vs. COPD vs. controls, 
Table II. Peripheral blood immune profiles of patients with COPD, patients with AECOPD and healthy controls.

\begin{tabular}{|c|c|c|c|c|}
\hline Immune profiles & $\begin{array}{c}\text { COPD } \\
(\text { mean } \pm \text { SEM })\end{array}$ & $\begin{array}{c}\text { AECOPD } \\
(\text { mean } \pm \text { SEM })\end{array}$ & $\begin{array}{r}\text { Healthy control } \\
(\text { mean } \pm \text { SEM })\end{array}$ & P-value \\
\hline \multicolumn{5}{|l|}{ Immune cells ${ }^{\mathrm{a}}, \%$} \\
\hline $\mathrm{CD}^{+} \mathrm{T}$ cell & $14.01 \pm 1.08$ & $11.90 \pm 0.9$ & $20.11 \pm 2.04^{\mathrm{b}, \mathrm{c}}$ & $0.001^{\mathrm{e}}$ \\
\hline $\mathrm{CD}^{+} \mathrm{CD}^{+} \mathrm{T}$ cell & $9.04 \pm 0.53^{c}$ & $6.50 \pm 0.89^{b}$ & $12.36 \pm 1.06^{\mathrm{b}, \mathrm{c}}$ & $0.001^{\mathrm{e}}$ \\
\hline $\mathrm{CD}^{+} \mathrm{CD}^{+}{ }^{+} \mathrm{T}$ cell & $5.55 \pm 0.36$ & $4.70 \pm 0.47$ & $7.85 \pm 0.97^{\mathrm{b}, \mathrm{c}}$ & 0.004 \\
\hline $\mathrm{CD} 4 / \mathrm{CD} 8$ & $1.97 \pm 0.13^{\mathrm{c}}$ & $1.47 \pm 0.21^{\mathrm{b}}$ & $1.89 \pm 0.17$ & 0.160 \\
\hline $\mathrm{CD}^{+}{ }^{+} \mathrm{CD} 4 \mathrm{CD}^{-} \gamma \delta \mathrm{T}$ cell & $1.13 \pm 0.17$ & $1.46 \pm 0.45$ & $1.40 \pm 0.23$ & 0.586 \\
\hline $\mathrm{CD}^{+} \mathrm{CD}^{2} 6^{+} \mathrm{NKT}$ & $2.88 \pm 0.24$ & $3.01 \pm 0.32$ & $2.93 \pm 0.48$ & 0.976 \\
\hline $\mathrm{CD}^{-\mathrm{CD} 56^{+} \mathrm{NK}}$ & $5.85 \pm 0.42$ & $5.90 \pm 0.71$ & $6.27 \pm 0.76$ & 0.877 \\
\hline CD19+ B cell & $2.11 \pm 0.25^{\mathrm{c}}$ & $1.69 \pm 0.20^{\mathrm{b}}$ & $2.01 \pm 0.34$ & 0.723 \\
\hline $\mathrm{CD}_{6}{ }^{+} \mathrm{CD} 16^{+} \mathrm{NEU}$ & $67.82 \pm 1.29$ & $68.21 \pm 2.6$ & $59.36 \pm 1.18^{\mathrm{b}, \mathrm{c}}$ & 0.011 \\
\hline $\mathrm{CD}_{14}^{+} \mathrm{MON}$ & $4.19 \pm 0.26$ & $4.57 \pm 0.33$ & $3.04 \pm 0.41^{\mathrm{b}, \mathrm{c}}$ & 0.027 \\
\hline $\mathrm{CD}^{+} 4^{+} \mathrm{MON}$ & $3.94 \pm 0.26$ & $4.53 \pm 0.53$ & $2.85 \pm 0.37^{\mathrm{b}, \mathrm{c}}$ & 0.026 \\
\hline $\mathrm{CD}_{14}{ }^{+} \mathrm{CD} 64^{+}$ & $3.19 \pm 0.23$ & $3.82 \pm 0.38$ & $2.53 \pm 0.37^{\mathrm{c}}$ & 0.070 \\
\hline \multicolumn{5}{|c|}{ Inflammatory factors, $\mathrm{pg} / \mathrm{ml}$ (by multi-plex panel) } \\
\hline IL-6 & $6.95 \pm 1.16$ & $20.51 \pm 3.59^{\mathrm{b}, \mathrm{d}}$ & $3.82 \pm 1.14^{\mathrm{b}, \mathrm{c}}$ & $0.001^{\mathrm{e}}$ \\
\hline TNF- $\alpha$ & $11.23 \pm 1.79$ & $21.71 \pm 3.31^{\mathrm{b}, \mathrm{d}}$ & $8.56 \pm 2.09^{\mathrm{c}}$ & 0.023 \\
\hline $\mathrm{IL}-1 \beta$ & $6.69 \pm 1.33$ & $7.24 \pm 1.20$ & $4.09 \pm 1.00^{\mathrm{c}}$ & 0.044 \\
\hline IFN- $\gamma$ & $11.91 \pm 1.80$ & $29.1 \pm 10.79^{\mathrm{b}, \mathrm{d}}$ & $10.32 \pm 1.45^{\mathrm{c}}$ & 0.032 \\
\hline IL-8 & $17.58 \pm 3.71$ & $53.39 \pm 14.61^{\mathrm{b}, \mathrm{d}}$ & $12.87 \pm 3.11^{\mathrm{c}}$ & $0.008^{\mathrm{e}}$ \\
\hline IL-33 & $11.10 \pm 2.27$ & $25.56 \pm 11.17^{\mathrm{d}}$ & $6.11 \pm 1.98^{\mathrm{c}}$ & 0.029 \\
\hline IL-17A & $4.57 \pm 0.60$ & $9.56 \pm 0.89^{\mathrm{b}, \mathrm{d}}$ & $4.15 \pm 1.02^{\mathrm{c}}$ & 0.047 \\
\hline IL-4 & $18.58 \pm 3.29$ & $12.23 \pm 1.28$ & $17.08 \pm 2.55$ & 0.595 \\
\hline IL-5 & $12.18 \pm 1.04$ & $12.71 \pm 1.13$ & $11.5 \pm 0.6$ & 0.843 \\
\hline IL-13 & $19.27 \pm 0.87$ & $20.27 \pm 1.71$ & $17.15 \pm 1.92$ & 0.296 \\
\hline TGF- $\alpha$ & $2.15 \pm 0.57$ & $2.20 \pm 0.73$ & $1.46 \pm 0.25^{\mathrm{b}, \mathrm{c}}$ & 0.006 \\
\hline GRO- $\alpha$ & $223.3 \pm 33.5^{\mathrm{c}}$ & $382.7 \pm 135 \cdot 4^{b}$ & $323.1 \pm 78.1$ & 0.065 \\
\hline SP-D & $6671 \pm 926.7$ & $7122 \pm 2273$ & $7767 \pm 1948$ & 0.800 \\
\hline MPO & $8856 \pm 843.8$ & $7201 \pm 1084$ & $8911 \pm 1156$ & 0.405 \\
\hline MMP-9 & $10627 \pm 1901$ & $6852.9 \pm 1367$ & $7019.7 \pm 905$ & 0.377 \\
\hline \multicolumn{5}{|c|}{ Inflammatory factors, pg/ml (by ELISA) } \\
\hline $\mathrm{CC} 16$ & $18.77 \pm 1.31^{\mathrm{c}}$ & $34.03 \pm 12.43^{\mathrm{b}}$ & $19.43 \pm 3.76$ & 0.342 \\
\hline Fibrinogen & $841.2 \pm 55.65$ & $716.4 \pm 76.07$ & $778.7 \pm 71.67$ & 0.211 \\
\hline $\mathrm{NE}$ & $499.3 \pm 80.34$ & $742.5 \pm 267.1$ & $634.8 \pm 178.6$ & 0.432 \\
\hline CRP & $1882 \pm 412.4$ & $4395 \pm 915.9^{\mathrm{b}, \mathrm{d}}$ & $2020 \pm 958 \cdot 6^{c}$ & 0.010 \\
\hline TGF- $\beta$ & $40.61 \pm 3.8$ & $39.51 \pm 7.53$ & $55.44 \pm 10.00$ & 0.193 \\
\hline
\end{tabular}

${ }^{\mathrm{a}} \mathrm{P}<0.05$ vs. human leucocytes $\left(\mathrm{CD} 45^{+}\right) .{ }^{\mathrm{b}} \mathrm{P}<0.05$ vs. $\mathrm{COPD} ;{ }^{\mathrm{c}} \mathrm{P}<0.05$ vs. AECOPD; ${ }^{\mathrm{d}} \mathrm{P}<0.05$ vs. control. ${ }^{\mathrm{e}} \mathrm{P}<0.001$. COPD, chronic obstructive pulmonary disorder; AE, acute exacerbated; NKT, natural killer T; NK, natural killer; NEU, neutrophil; MON, monocyte; GRO- $\alpha$, human growth-regulated oncogene $\alpha$; SP-D, pulmonary surfactant-associated protein D; MPO, myeloperoxidase; CC16, club cell secretory protein; NE, neutrophil elastase.

29.10 vs. 11.91 vs. 10.32), IL-8 (AECOPD vs. COPD vs. controls, 53.39 vs. 17.58 vs. 12.87), IL-17A (AECOPD vs. COPD vs. controls, 9.56 vs. 4.57 vs. 4.15) and CRP (AECOPD vs. COPD vs. controls, 4395 vs. 1882 vs. 2020) were markedly increased in the AECOPD group compared with the COPD group and healthy controls. IL-33 levels were higher in the AECOPD group compared with healthy controls (AECOPD vs. controls, 25.56 vs. 6.11), while GRO- $\alpha$ was higher in the AECOPD group compared with the COPD group (AECOPD vs. COPD, 382.7 vs.
223.3). The other parameters exhibited no significant differences among the groups (Table II; Fig. 4).

Bioinformatics analysis. Proteomic interaction analysis of key biomarkers was performed by inputting 10 significant biomarkers (IL-6, IL-1 $\beta$, TNF- $\alpha$, IFN- $\gamma$, IL-8, IL-33, IL-17A, TGF- $\alpha$, GRO- $\alpha$ and CRP) into the STRING database. The results indicated that there were three core biomarkers of COPD: IL-6, TNF- $\alpha$ and IL-8. In addition, IFN- $\gamma$, CRP, GRO- $\alpha$, IL-33 
Table III. Peripheral blood immune cells of smoking and non-smoking subjects.

\begin{tabular}{|c|c|c|c|c|c|}
\hline Characteristics & $\begin{array}{c}\text { Smoking } \\
\text { COPD }(n=73)\end{array}$ & $\begin{array}{l}\text { Non-smoking } \\
\text { COPD }(n=24)\end{array}$ & $\begin{array}{c}\text { Smoking } \\
\text { control }(n=8)\end{array}$ & $\begin{array}{l}\text { Non-smoking } \\
\text { control }(n=17)\end{array}$ & P-value \\
\hline Age, years (mean \pm SEM) & $65.81 \pm 0.96$ & $64.27 \pm 2.04$ & $63.18 \pm 2.94$ & $65.15 \pm 1.23$ & 0.715 \\
\hline Male, n (\%) & $68(93.15)$ & $8(33.33)$ & $8(100.00)$ & $7(41.18)$ & $0.001^{\mathrm{a}}$ \\
\hline Pack-years (mean \pm SEM) & $40.78 \pm 2.88$ & & $31.56 \pm 7.02$ & & 0.327 \\
\hline $\mathrm{CD}^{+} \mathrm{T}$ cell $($ mean $\pm \mathrm{SEM})$ & $12.88 \pm 0.65$ & $15.43 \pm 1.67$ & $15.82 \pm 2.57$ & $22.25 \pm 2.66^{\mathrm{b}-\mathrm{d}}$ & $0.001^{\mathrm{a}}$ \\
\hline $\mathrm{CD}^{+}{ }^{+} \mathrm{CD} 4^{+} \mathrm{T}$ cell $($ mean $\pm \mathrm{SEM})$ & $8.16 \pm 0.48$ & $9.42 \pm 1.24$ & $11.58 \pm 1.95$ & $12.75 \pm 1.29^{\mathrm{b}, \mathrm{c}}$ & 0.003 \\
\hline $\mathrm{CD}^{+} \mathrm{CD}^{+} \mathrm{T}$ cell $($ mean $\pm \mathrm{SEM})$ & $5.15 \pm 0.31$ & $5.98 \pm 0.74$ & $5.33 \pm 1.01$ & $9.11 \pm 1.30^{\mathrm{b}-\mathrm{d}}$ & $0.001^{\mathrm{a}}$ \\
\hline CD4/CD8 (mean \pm SEM) & $1.89 \pm 0.12$ & $1.82 \pm 0.16$ & $2.45 \pm 0.40$ & $1.61 \pm 0.13$ & 0.296 \\
\hline $\mathrm{CD}^{+}{ }^{+} \mathrm{CD} 4{ }^{-} \mathrm{CD}^{-}($mean $\pm \mathrm{SEM})$ & $1.14 \pm 0.19$ & $1.41 \pm 0.33$ & $1.06 \pm 0.31$ & $1.57 \pm 0.30$ & 0.675 \\
\hline $\mathrm{CD}^{+}{ }^{+} \mathrm{CD}_{56}{ }^{+} \mathrm{NKT}($ mean $\pm \mathrm{SEM})$ & $2.91 \pm 0.24$ & $2.87 \pm 0.39$ & $2.41 \pm 0.49$ & $3.19 \pm 0.68$ & 0.854 \\
\hline $\mathrm{CD}^{-}{ }^{-} \mathrm{CD}^{+} 6^{+} \mathrm{NK}($ mean $\pm \mathrm{SEM})$ & $6.26 \pm 0.42$ & $4.71 \pm 0.64$ & $5.82 \pm 1.41$ & $6.50 \pm 0.93$ & 0.268 \\
\hline $\mathrm{CD} 19^{+} \mathrm{B}$ cell $($ mean $\pm \mathrm{SEM})$ & $1.74 \pm 0.13^{\mathrm{c}}$ & $2.41 \pm 0.34^{\mathrm{b}}$ & $1.58 \pm 0.63$ & $2.23 \pm 0.41$ & 0.125 \\
\hline $\mathrm{CD} 66^{+} \mathrm{CD} 16^{+} \mathrm{NEU}($ mean $\pm \mathrm{SEM})$ & $68.83 \pm 1.24$ & $64.99 \pm 2.73$ & $66.41 \pm 2.85$ & $56.04 \pm 4.29^{\mathrm{b}-\mathrm{d}}$ & 0.002 \\
\hline $\mathrm{CD}_{14}^{+} \mathrm{MON}($ mean $\pm \mathrm{SEM})$ & $4.42 \pm 0.27$ & $3.85 \pm 0.25$ & $4.05 \pm 0.69$ & $2.56 \pm 0.47^{\mathrm{b}-\mathrm{d}}$ & 0.014 \\
\hline $\mathrm{CD}^{+} 4^{+} \mathrm{MON}($ mean $\pm \mathrm{SEM})$ & $4.18 \pm 0.27$ & $3.75 \pm 0.48$ & $3.96 \pm 0.73$ & $2.32 \pm 0.37^{\mathrm{b}-\mathrm{d}}$ & 0.020 \\
\hline $\mathrm{CD} 14^{+} \mathrm{CD}^{+} 4^{+}($mean $\pm \mathrm{SEM})$ & $3.49 \pm 0.24$ & $2.86 \pm 0.32^{b}$ & $3.58 \pm 0.66$ & $2.03 \pm 0.40^{\mathrm{b}, \mathrm{d}}$ & 0.029 \\
\hline
\end{tabular}

${ }^{\mathrm{a}} \mathrm{P}<0.001 .{ }^{\mathrm{b}} \mathrm{P}<0.05$ vs. smoking COPD group; ${ }^{\mathrm{c}} \mathrm{P}<0.05$ vs. non-Smoking COPD; ${ }^{\mathrm{d}} \mathrm{P}<0.05$ vs. smoking control group. COPD, chronic obstructive pulmonary disorder; NKT, natural killer T; NK, natural killer; NEU, neutrophil; MON, monocyte.

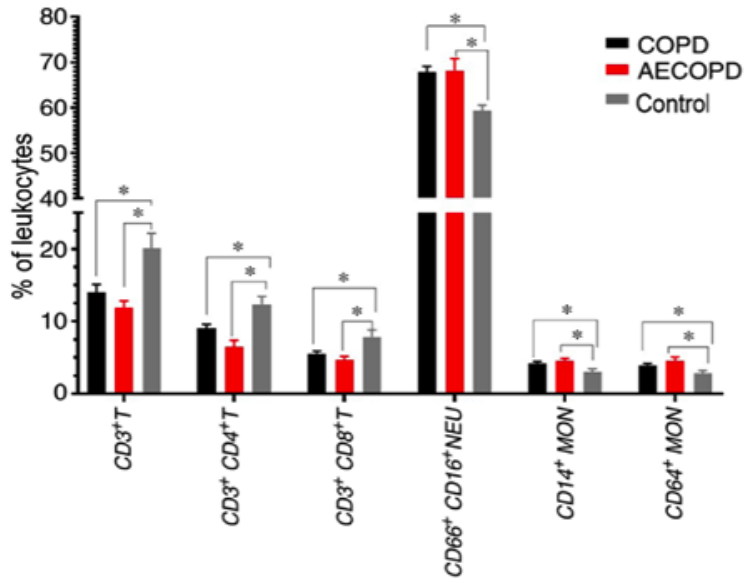

Figure 3. Proportions (\%) of immune cell expression in patients with COPD, patients with AECOPD and healthy controls. ${ }^{*} \mathrm{P}<0.05$. COPD, chronic obstructive pulmonary disorder; AE, acute exacerbated; MON, monocyte; NEU, neutrophil.

and IL-17A were also essential in the complex network (Fig. 5). The present study used network expansion function through STRING and found some potential genes closely associated with these proteins, including inhibitor of $\mathrm{NF}-\kappa \mathrm{B}$ kinase regulatory subunit $\gamma$ (IKBKG), inhibitor of kappa light polypeptide gene enhancer in B-cells, kinase $\beta$ (IKBKB), receptor-interacting serine/threonine kinase 1 (RIPK1), TNF receptor superfamily member $1 \mathrm{~A}$ associated via death domain (TRADD) and TNF receptor-associated factor 2 (TRAF2; Fig. 6).

Correlation analysis. Pearson's correlation analysis revealed a mild positive correlation between $\mathrm{CD} 3^{+} \mathrm{T}$ lymphocytes (mainly CD4 subset) with FEV1/FVC (r=0.33), FEV1\% $(\mathrm{r}=0.27)$ and maximal mid expiratory flow (MMEF)75/25 $(r=0.34)$ parameters; however, Spearman's correlation analysis revealed a mild or weak negative correlation with presence of smoking and exacerbation history. Myeloid-derived immune cells, such as $\mathrm{CD}^{+} 6^{+} \mathrm{CD}^{+} 6^{+}$neutrophil $(\mathrm{r}=-0.26, \mathrm{r}=-0.25)$ and $\mathrm{CD}_{4}{ }^{+}(\mathrm{r}=-0.22, \mathrm{r}=-0.24) / \mathrm{CD}^{+} 4^{+}(\mathrm{r}=-0.14, \mathrm{r}=-0.22) \mathrm{MON}$ exhibited a weak negative correlation with pulmonary function (FEV1/FVC, MMEF75/25). In addition, MMP-9 exhibited a mild negative correlation with cough $(\mathrm{r}=-0.33)$ but was not correlated with spirometry measurements. Furthermore, IL-8 $(r=0.33)$ and IL-17A $(r=0.31)$ exhibited a mild positive correlation with cough, while CRP was mildly negatively correlated with FEV1/FVC ( $r=-0.21)$, FEV1\% ( $r=-0.24)$ and MMEF75/25 $(\mathrm{r}=-0.26)$, smoking history $(\mathrm{r}=-0.62)$ but mild positively correlated with exacerbations $(\mathrm{r}=0.26), \mathrm{mMRC}(\mathrm{r}=0.26)$ and cough $(\mathrm{r}=0.28)$. Additionally, $\mathrm{CC} 16$ exhibited a moderate negative correlation with TLC (total lung compacity) ( $\mathrm{r}=-0.44)$ and RV (residual volume)/TLC ( $\mathrm{r}=-0.41$; Tables IV and $\mathrm{V}$; all $\mathrm{P}<0.05$; correlation strength: $r=0.6-0.8$ strong; $r=0.4-0.6$ moderate; $\mathrm{r}=0.2-0.4$ mild and $\mathrm{r}=0.0-0.2$ weak or uncorrelated).

\section{Discussion}

Inflammation serves a central role in the activation and alteration of immune profiles in COPD (26). In the present study, significant differences in the expression profiles of immune factors in peripheral blood of patients with COPD were observed, based on results from flow cytometry and analysis of multiple biomarker panels. Specifically, there was a decrease in $\mathrm{CD}^{+} \mathrm{T}$ lymphocytes and an increase in $\mathrm{CD} 14^{+} / \mathrm{CD} 64^{+} \mathrm{MPS}$ and $\mathrm{CD}^{+} 6^{+} \mathrm{CD}^{+} 6^{+}$neutrophils in both the COPD/AECOPD groups and smoking controls compared with non-smoking controls. In addition, alterations of systemic inflammatory markers, such as IL-6, TNF- $\alpha$, IFN- $\gamma$, IL-17A and CRP, were reported, suggesting 

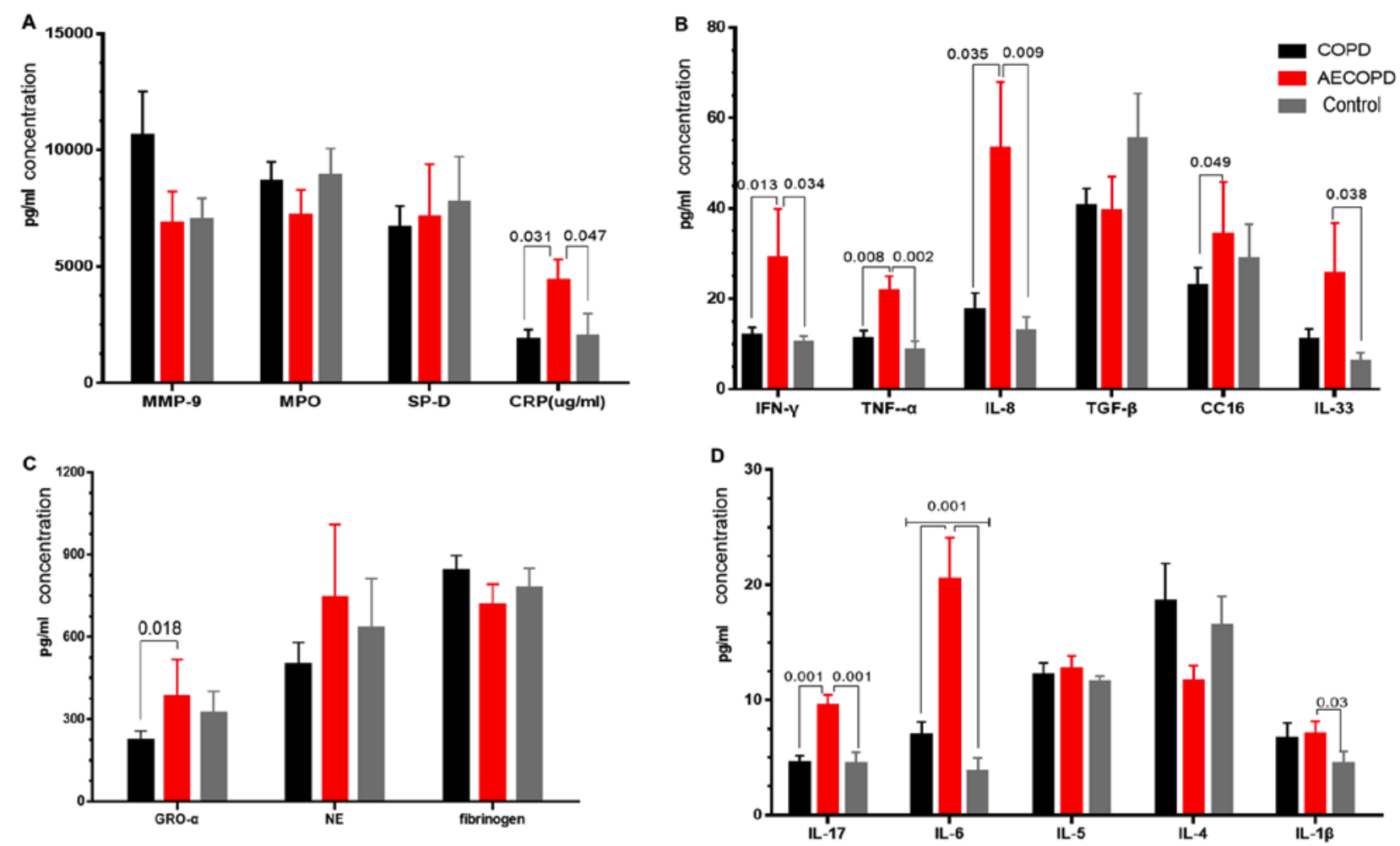

Figure 4. Levels of inflammatory factors in patients with COPD, patients with AECOPD and healthy controls. (A) Levels of CRP were increased in the AECOPD group. (B) Levels of TNF- $\alpha$, IFN- $\gamma$ and IL-8 were increased in the AECOPD group. (C) Levels of GRO- $\alpha$ were increased in the AECOPD group compared with COPD. (D) Levels of IL-6 and IL-17A were increased in the AECOPD group. Data were analyzed using the Kruskal Wallis test and Dunn's post hoc test. COPD, chronic obstructive pulmonary disorder; AE, acute exacerbated; MPO, myeloperoxidase; SP-D, pulmonary surfactant-associated protein D; $\mathrm{CC} 16$, club cell secretory protein; GRO- $\alpha$, human growth-regulated oncogene $\alpha$; NE, neutrophil elastase.

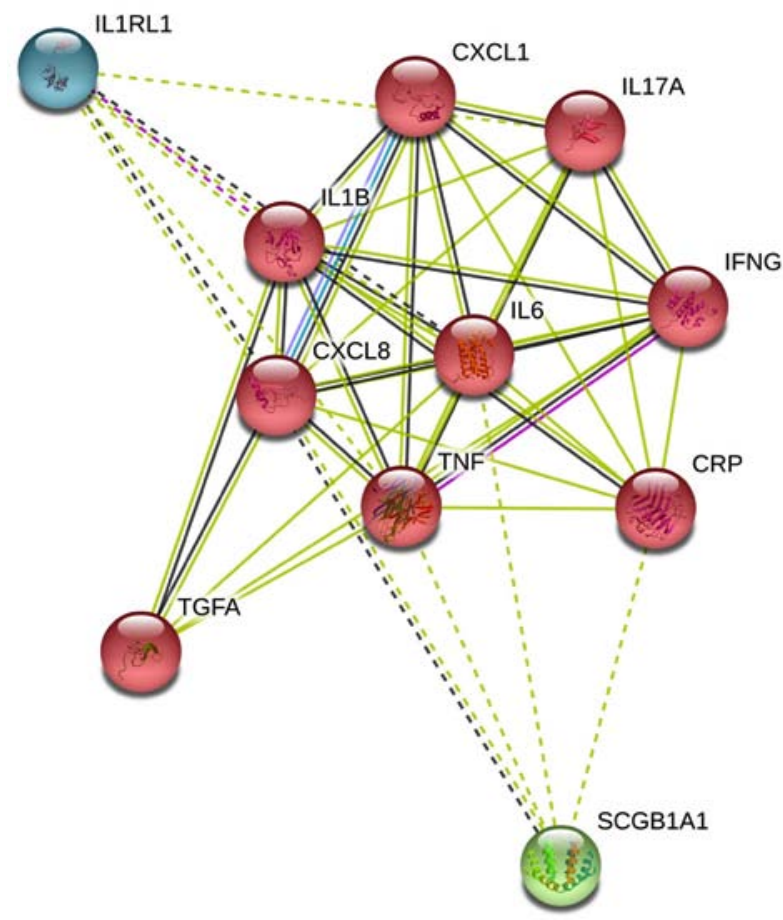

Figure 5. Protein-protein interaction network of significant inflammatory factors (IL-6, IL-1 $\beta$, TNF- $\alpha$, IFN- $\gamma$, IL-8, IL-33, IL-17A, TGF- $\alpha$, GRO- $\alpha$ and CRP). Network nodes represent proteins (colored nodes: Query proteins and first shell of interactors), while edges represent protein-protein associations [from STRING website, 10 items (human)-STRING interaction network (string-db.org)]. IL-6, TNF- $\alpha$ and IL-8 were predominant in the network of significantly different inflammatory factors (triangle).

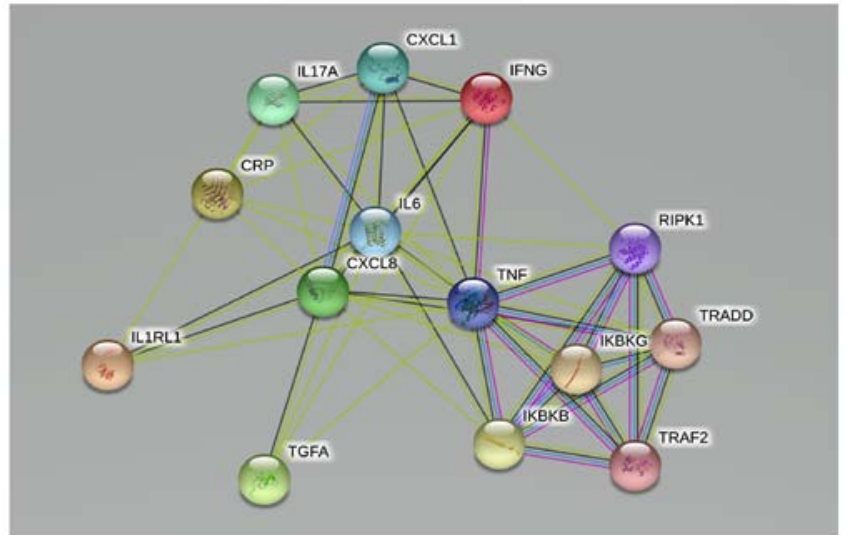

Figure 6. Expansion of protein-protein interaction network of significant inflammatory factors. Network-associated proteins IKBKG, IKBKB, RIPK1, TRADD and TRAF2 (expanded search) were closely associated with other factors. IKBKG, including inhibitor of NF- $\mathrm{KB}$ kinase regulatory subunit $\gamma$; IKBKB, inhibitor of kappa light polypeptide gene enhancer in B-cells, kinase $\beta$; RIPK1, receptor-interacting serine/threonine kinase 1; TRADD, TNF receptor superfamily member $1 \mathrm{~A}$ associated via death domain; TRAF2, TNF receptor-associated factor 2.

that immune profiles were different among patients with COPD, patients with AECOPD and healthy controls, CRP and IL-17A were associated with smoking status.

A previous study reported an increase in the proportion of macrophages (M1-M2) and a decrease in T-lymphocytes (mainly $\mathrm{CD} 4^{+} \mathrm{T}$ ) in lung tissues of current smokers with COPD 
Table IV. Pearson correlation coefficient between immune profiles and clinical features.

\begin{tabular}{|c|c|c|c|c|c|c|}
\hline Correlation (r) & FEV1/FVC & FEV1 & $\mathrm{FVC}$ & RV/TLC & TLC & MM75/25 \\
\hline $\mathrm{CD}^{+} \mathrm{T}$ cell & 0.33 & 0.27 & 0.14 & 0.13 & -0.10 & 0.34 \\
\hline $\mathrm{CD}^{+} \mathrm{T}$ cell & 0.35 & 0.28 & 0.24 & -0.01 & -0.15 & 0.38 \\
\hline $\mathrm{CD}^{+} \mathrm{T}$ cell & 0.24 & 0.13 & 0.05 & 0.19 & 0.02 & 0.23 \\
\hline $\mathrm{CD}_{14}^{+} \mathrm{MPS}$ & -0.22 & -0.24 & -0.19 & -0.07 & -0.01 & -0.24 \\
\hline $\mathrm{CD}^{+} 4^{+} \mathrm{MPS}$ & -0.14 & -0.12 & -0.10 & -0.12 & -0.47 & -0.22 \\
\hline $\mathrm{CD} 16^{+} \mathrm{CD} 66^{+}$ & -0.26 & -0.20 & -0.01 & -0.17 & 0.06 & -0.25 \\
\hline IL-6 & -0.19 & -0.15 & 0.20 & 0.02 & 0.11 & -0.14 \\
\hline CRP & -0.21 & -0.24 & -0.23 & -0.04 & 0.02 & -0.26 \\
\hline CC16 & -0.03 & -0.02 & -0.07 & -0.41 & -0.44 & -0.05 \\
\hline IL-8 & -0.09 & -0.08 & -0.18 & -0.17 & 0.21 & -0.07 \\
\hline IL-17A & -0.24 & -0.31 & -0.28 & -0.44 & 0.41 & -0.26 \\
\hline Fibrinogen & -0.05 & -0.04 & -0.01 & -0.13 & -0.13 & -0.11 \\
\hline
\end{tabular}

MPS, mononuclear phagocyte system; CC16, club cell secretory protein; FEV1, forced expiratory volume in 1 sec; FVC, forced vital capacity; $\mathrm{RV}$, residual volume; TLC, total lung compacity; MMEF75/25, maximal mid expiratory flow.

Table V. Spearman's correlation coefficient between immune profiles and clinical features.

\begin{tabular}{|c|c|c|c|c|}
\hline Correlation coefficient & Smoking & Exacerbation & mMRC & Cough \\
\hline $\mathrm{CD}^{+} \mathrm{T}$ cell & $-0.29^{\mathrm{a}}$ & $-0.24^{\mathrm{a}}$ & -0.04 & 0.06 \\
\hline $\mathrm{CD}^{4}{ }^{+} \mathrm{T}$ cell & $-0.20^{\mathrm{a}}$ & -0.21 & -0.17 & 0.03 \\
\hline $\mathrm{CD}^{+} \mathrm{T}$ cell & $-0.23^{\mathrm{a}}$ & -0.15 & 0.20 & -0.05 \\
\hline $\mathrm{CD} 14^{+} \mathrm{MPS}$ & $0.27^{\mathrm{a}}$ & 0.16 & 0.13 & -0.04 \\
\hline $\mathrm{CD}^{+} 4^{+} \mathrm{MPS}$ & $0.31^{\mathrm{a}}$ & 0.10 & 0.10 & -0.07 \\
\hline $\mathrm{CD} 16^{+} \mathrm{CD} 66^{+}$ & $0.24^{\mathrm{a}}$ & -0.09 & -0.06 & -0.07 \\
\hline IL-6 & 0.15 & 0.22 & 0.16 & $0.24^{\mathrm{a}}$ \\
\hline CRP & -0.62 & $0.26^{\mathrm{a}}$ & $0.26^{\mathrm{a}}$ & $0.28^{\mathrm{a}}$ \\
\hline CC16 & -0.04 & 0.16 & 0.18 & -0.13 \\
\hline IL-8 & 0.12 & 0.14 & 0.20 & $0.33^{\mathrm{a}}$ \\
\hline IL-17A & -0.24 & $0.30^{\mathrm{a}}$ & 0.14 & $0.31^{\mathrm{a}}$ \\
\hline Fibrinogen & 0.07 & -0.24 & -0.21 & -0.20 \\
\hline MMP-9 & 0.07 & 0.02 & -0.06 & $-0.33^{\mathrm{a}}$ \\
\hline
\end{tabular}

${ }^{\mathrm{a}} \mathrm{P}<0.005$. MPS, mononuclear phagocyte system; $\mathrm{CC} 16$, club cell secretory protein; mMRC, modified Medical Research Council dyspnea scale.

compared with non-smokers and smokers without COPD (27). Furthermore, the proportion of monocytes was markedly different between non-smokers and smokers in the blood only (27). The present study reported an increase in the proportion of $\mathrm{CD} 16^{+} \mathrm{CD} 66^{+}$neutrophils and $\mathrm{CD} 14^{+} / \mathrm{CD} 64^{+} \mathrm{MPS}$, a decrease in the proportion of $\mathrm{CD}^{+} \mathrm{T}$ lymphocytes (both $\mathrm{CD}^{+}$and $\mathrm{CD}^{+} \mathrm{T}$ Cell) in patients with COPD and smokers compared with non-smoking controls in peripheral blood. This indicated the predominant effects of smoking and cumulative immune composition changes of COPD.

MPS is defined as a cell lineage, including promonocytes and their precursors, in the bone marrow, monocytes in circulation and macrophages in tissues (28). Once in the blood, monocytes undergo transformation into tissue macrophages with phagocytic function, and serve a central role in the immune regulation by generating antigens to $\mathrm{T}$ lymphocytes and secreting proinflammatory factors such as TNF $\alpha$, IL- $1 \beta$ and IL-6 that are involved in host defense and inflammation $(28,29)$. Although the present study did not assess macrophages in lung tissues and sputum, the increased ratio of $\mathrm{CD} 14^{+} / \mathrm{CD} 64^{+}$monocytes and $\mathrm{CD}^{+} 6^{+} \mathrm{CD}^{+} 6^{+}$neutrophils in blood revealed the prominent systemic inflammation of smokers and patients with COPD, and the decreased ratio of $\mathrm{CD}^{+} \mathrm{T}$ lymphocytes $\left(\mathrm{CD}^{+}\right.$and $\mathrm{CD}^{+}$ subsets) in the blood revealed the cumulative immune deficiency of smokers and patients with COPD. Lower proportions of lymphocytes $\left(\mathrm{CD}^{+} \mathrm{T}\right.$ cells) indicated poorer lung function and a higher risk of exacerbation in COPD. On the other hand, higher levels of myeloid cells $\left(\mathrm{CD} 16^{+} \mathrm{CD} 66^{+}\right.$neutrophils and 
$\left.\mathrm{CD} 14^{+} / \mathrm{CD} 4^{+} \mathrm{MPS}\right)$ indicated poorer lung function in smokers with COPD compared with non-smoking controls. However, the trigger of immune composition changes in non-smoking patients with COPD remains unclear.

Blood biomarkers have a significant value in diagnosis and prognosis of COPD (14). A study on COPD Gene and SPIROMCIS measured 114 candidate plasma and serum biomarkers using the 13 panel Luminex assays (30). A total of nine cytokines and chemokines were selected for further analysis using a Meso Scale Discovery platform. These MSD multiplex panels were used to measure Single nucleotide polymorphism in 2,123 subjects from COPD Gene and 1,117 subjects from SPIROMICS. The results indicated a strong association between eotaxin and IL-6 with airflow obstruction (13). In the present multiplex panel involving 20 biomarkers, it was identified that IL-6, TNF- $\alpha$ and IL-8 (CXCL-8) served key roles in the inflammation response network of COPD. Pearson's correlation analysis revealed a close relationship between CRP and airway airflow obstruction, while CC16 could be a novel marker of emphysema. Furthermore, higher levels of IL-6, CRP, IL-8 and IL-17A indicated more cough, sputum and dyspnea. Although no biomarker has been demonstrated to be useful in the diagnosis of COPD to date, to the best of our knowledge, blood-based biomarkers for predicting progression of COPD remain relevant (31). The present findings may provide novel insights into biomarkers that have potential for prediction and evaluation of COPD pulmonary function, as well as its symptoms, and are expected to be beneficial for development of novel therapies for COPD phenotypes.

The present study analyzed the interaction among biomarkers, identified the importance of IKBKG, RIPK1, TRADD and TRAF2 and also investigated IL-17, TNF and NF- $\kappa$ B pathway, which are associated with inflammatory cell chemotaxis and immune response (25). The IL-17A, TNF and NF- $\kappa \mathrm{B}$ signaling pathways have been identified to be important in the inflammatory response of COPD (32). Fisetin, which inhibits the TNF- $\alpha$ and NF- $\mathrm{NB}$ signaling pathways, could be a good candidate drug for improving lung function in patients with COPD (33). In addition, IL-17A-driven type-2 inflammation is another endotype of COPD, which could be an indicator of steroid-unresponsive subgroup of COPD (34).

Previous studies have found T helper 17 (Th17) cells, a subset of CD4 ${ }^{+} \mathrm{T}$ cells, to be positively correlated with IL-17A levels, which are increased in patients with COPD (35). The present study reported elevated levels of IL-17A, although the proportion of $\mathrm{CD}^{+} \mathrm{T}$ cells was decreased in patients with COPD compared with healthy controls. A possible reason for this paradox is that Th17 cells could be inhibiting the expansion of $\mathrm{CD} 4^{+}$regulatory T (Treg) cells (36). An imbalance in Th17/Treg has been reported to serve a pivotal role in COPD development and progression (37). Studies have also demonstrated a negative correlation between Th17 cell and Treg cells in patients with COPD $(34,38)$. It is possible that the lower proportion of Treg cells could have contributed to the decrease of $\mathrm{CD}^{+} \mathrm{T}$ cells in COPD in the present study. Furthermore, a positive correlation between Treg proportion and TGF- $\beta$ level has been reported (38). However, the present study did not observe a significant decrease of TGF- $\beta$ in COPD. Further functional experiments investigating the subset of immune cells are required to investigate this.

The present study had several limitations. The wide range of immune cells should be classified into more detailed subgroups.
The functional and dynamic evolution of various immune components at different stages will need to be explored in future studies. The biomarker panel used was selected from available assays, based on possible mechanisms in COPD. This panel was heavily weighted towards systemic inflammatory markers, not lung-specific biomarkers. In addition, a larger population of participants would be helpful in identifying subgroups to help decrease the heterogeneity. Despite these limitations, the present study identified COPD and smoking associated immune profiles, and indicated those relevant to pulmonary functions and symptoms. Potential pathways and genes of the inflammatory molecular network were also identified.

Overall, the present study revealed changes in the immune profiles in patients with COPD and smokers. A decrease in $\mathrm{CD}^{+} \mathrm{T}$ cells and an increase in neutrophils and MPS were also reported. In addition, levels of IL-6, TNF- $\alpha$, IFN- $\gamma$, IL-8 IL-17A and CRP were higher in patients with AECOPD compared with patients with COPD and healthy controls. Furthermore, IL-6, TNF- $\alpha$ and IL-8 (CXCL8) were identified as core biomarkers in COPD pathogenesis, while immune profiles were also relevant to pulmonary functions and symptoms. Future studies will need to recruit more patients with COPD in order to validate and expand the results. Particular focus should be paid on investigating lung-specific biomarkers to identify more COPD subgroups and help advance novel therapies for COPD.

\section{Acknowledgements}

The authors thank Professor Dan Liu and Professor Gang Wang of West China Hospital, Chengdu, China, for assistance with the patients enrollment and experiments.

\section{Funding}

The study was supported by The National Key Development Plan for Precision Medicine Research of China (grant no. 2017YFC0910004).

\section{Availability of data and materials}

All data generated or analyzed during this study are included in this published article.

\section{Authors' contributions}

SL and SZ conceived the present study, enrolled the subjects, and recorded all the clinical data. $\mathrm{ZW}$ assisted in the testing of inflammatory factors. FW performed the flow cytometry. WL organized the study, supervised the study and was involved in data aquisition, analysis and interpretation. SL and SZ confirm the authenticity of all the raw data. All authors reviewed and approved the final manuscript.

\section{Ethics approval and consent to participate}

The study was approved by The Ethics Committee of West China Hospital, Sichuan University (Chengdu, China) and all patients provided written informed consent. 


\section{Patient consent for publication}

Not applicable.

\section{Competing interests}

The authors declare that they have no competing interests.

\section{References}

1. GBD 2017 Causes of Death Collaborators: Global, regional, and national age-sex-specific mortality for 282 causes of death in 195 countries and territories, 1980-2017: A systematic analysis for the Global Burden of Disease Study 2017. Lancet 392: 1736-1788, 2018.

2. Singh D, Agusti A, Anzueto A, Barnes PJ, Bourbeau J, Celli BR Criner GJ, Frith P, Halpin DMG, Han M, et al: Global strategy for the diagnosis, management, and prevention of chronic obstructive lung disease: The GOLD science committee report 2019. Eur Respir J 53: 1900164, 2019.

3. Decramer M, Janssens W and Miravitlles M: Chronic obstructive pulmonary disease. Lancet 379: 1341-1351, 2012.

4. Tuder RM and Petrache I: Pathogenesis of chronic obstructive pulmonary disease. J Clin Invest 122: 2749-2755, 2012.

5. Brightling $\mathrm{C}$ and Greening N: Airway inflammation in COPD-progress to precision medicine. Eur Respir J 54: 1900651, 2019.

6. Gorska K, Paplinska-Goryca M, Nejman-Gryz P, Goryca K and Krenke R: Eosinophilic and neutrophilic airway inflammation in the phenotyping of mild-to-moderate asthma and chronic obstructive pulmonary disease. COPD 14: 181-189, 2017.

7. Magnussen H: Therapy control of COPD by eosinophilic granulocytes?. Dtsch Med Wochenschr 144: 917-921, 2019 (In German)

8. Pavord ID: Biologics and chronic obstructive pulmonary disease. J Allergy Clin Immunol 141: 1983-1991, 2018.

9. Yousuf A and Brightling CE: Biologic drugs: A new target therapy in COPD? COPD 15: 99-107, 2018.

10. Cruz T, Lopez-Giraldo A, Noell G, Casas-Recasens S, Garcia T, Molins L, Juan M, Fernandez MA, Agustí A and Faner R: Multi-level immune response network in mild-moderate chronic obstructive pulmonary disease (COPD). Respir Res 20: 152, 2019.

11. Pan Z, Yu H and Liao JL: Probing cellular and molecular mechanisms of cigarette smoke-induced immune response in the progression of chronic obstructive pulmonary disease using multiscale network modeling. PLoS One 11: e0163192, 2016.

12. Butler CC, Gillespie D, White P, Bates J, Lowe R, Thomas-Jones E, Wootton M, Hood K, Phillips R, Melbye H, et al: C-Reactive protein testing to guide antibiotic prescribing for COPD exacerbations. N Engl J Med 381: 111-120, 2019.

13. Bradford E, Jacobson S, Varasteh J, Comellas AP, Woodruff P, O'Neal W, DeMeo DL, Li X, Kim V, Cho M, et al: The value of blood cytokines and chemokines in assessing COPD. Respir Res 18: 180, 2017.

14. Regan EA, Hersh CP, Castaldi PJ, DeMeo DL, Silverman EK, Crapo JD and Bowler RP: Omics and the search for blood biomarkers in Chronic obstructive pulmonary disease: Insights from COPDGene. Am J Respir Cell Mol Biol 61: 143-149, 2019.

15. Zou Y, Chen X, Liu J, Zhou DB, Kuang X, Xiao J, Yu Q, Lu X, $\mathrm{Li}$ W, Xie B and Chen Q: Serum IL-1 $\beta$ and IL-17A levels in patients with COPD: Associations with clinical parameters. Int J Chron Obstruct Pulmon Dis 12: 1247-1254, 2017.

16. Fermont JM, Masconi KL, Jensen MT, Ferrari R, Di Lorenzo VAP, Marott JM, Schuetz P, Watz H, Waschki B, Müllerova H, et al Biomarkers and clinical outcomes in COPD: A systematic review and meta-analysis. Thorax 74: 439-446, 2019.

17. Bai Y, Zhou Q, Fang Q, Song L and Chen K: Inflammatory cytokines and T-Lymphocyte subsets in serum and sputum in patients with bronchial asthma and chronic obstructive pulmonary disease. Med Sci Monit 25: 2206-2210, 2019.

18. Papaioannou AI, Konstantelou E, Papaporfyriou A, Bartziokas K, Spathis A, Bakakos P, Loukides S, Koulouris N, Papiris S and Kostikas K: Serum surfactant protein levels in patients admitted to the hospital with acute COPD exacerbation.Lung 196: 201-205, 2018

19. Zhai J, Insel M, Addison KJ, Stern DA, Pederson W, Dy A, Rojas-Quintero J, Owen CA, Sherrill DL, Morgan W, et al: Club cell secretory protein deficiency leads to altered lung function. Am J Respir Crit Care Med 199: 302-312, 2019.
20. Rangasamy L, Geronimo BD, Ortin I, Coderch C, Zapico JM, Ramos A and de Pascual-Teresa B: Molecular imaging probes based on matrix metalloproteinase inhibitors (MMPIs). Molecules 24: 2982, 2019.

21. Stockley RA, Halpin DMG, Celli BR and Singh D: Chronic obstructive pulmonary disease Biomarkers and their interpretation. Am J Respir Crit Care Med 199: 1195-1204, 2018.

22. Graham BL, Steenbruggen I, Miller MR, Barjaktarevic IZ, Cooper BG, Hall GL, Hallstrand TS, Kaminsky DA, McCarthy K, McCormack MC, et al: Standardization of spirometry 2019 update. An official American thoracic society and European respiratory society technical statement. Am J Respir Crit Care Med 200: e70-e88, 2019.

23. Munari AB, Gulart AA, Dos Santos K, Venâncio RS, Karloh M and Mayer AF: Modified medical research council dyspnea scale in GOLD classification better reflects physical activities of daily living. Respir Care 63: 77-85, 2018.

24. Skierka AS and Michels KB: Ethical principles and placebo-controlled trials-interpretation and implementation of the Declaration of Helsinki's placebo paragraph in medical research. BMC Med Ethics 19: 24, 2018.

25. Szklarczyk D, Gable AL, Lyon D, Junge A, Wyder S, Huerta-Cepas J, Simonovic M, Doncheva NT, Morris JH, Bork P, et al: STRING v11: Protein-protein association networks with increased coverage, supporting functional discovery in genome-wide experimental datasets. Nucleic Acids Res 47: D607-D613, 2019.

26. Caramori G, Casolari P, Barczyk A, Durham AL, Di Stefano A and Adcock I: COPD immunopathology. Semin Immunopathol 38: 497-515, 2016

27. Cruz T, Lopez-Giraldo A, Noell G, Molins L, Juan M, Fernandez MA, Canet MRF and Agusti A: Pulmonary and systemic cellular immune response network in patients with mild-moderate COPD. Eur Respiratory J Conf 50: 2017.

28. Hume DA, Irvine KM and Pridans C: The mononuclear phagocyte system: The relationship between monocytes and macrophages. Trends Immunol 40: 98-112, 2019.

29. Yang J, Zhang L, Yu C, Yang XF and Wang H: Monocyte and macrophage differentiation: Circulation inflammatory monocyte as biomarker for inflammatory diseases. Biomark Res 2: 1, 2014.

30. Sun W, Kechris K, Jacobson S, Drummond MB, Hawkins GA Yang J, Chen TH, Quibrera PM, Anderson W, Barr RG, et al: Common genetic polymorphisms influence blood biomarker measurements in COPD. PLoS Genet 12: e1006011, 2016.

31. Mannino DM: Biomarkers for chronic obstructive pulmonary disease diagnosis and progression: Insights, disappointments and promise. Curr Opin Pulm Med 25: 144-149, 2019.

32. Garudadri S and Woodruff PG: Targeting chronic obstructive pulmonary disease phenotypes, endotypes, and biomarkers. Ann Am Thorac Soc 15 (Suppl 4): S234-S238, 2018.

33. Lee S, Ro H, In HJ, Choi JH, Kim MO, Lee J, Hong ST and Lee SU: Fisetin inhibits TNF- $\alpha / \mathrm{NF}-\kappa \mathrm{B}$-induced IL- 8 expression by targeting PKC $\delta$ in human airway epithelial cells. Cytokine 108: 247-254, 2018.

34. Christenson SA, van den Berge M, Faiz A, Inkamp K, Bhakta N, Bonser LR, Zlock LT, Barjaktarevic IZ, Barr RG, Bleecker ER, et al: An airway epithelial IL-17A response signature identifies a steroid-unresponsive COPD patient subgroup. J Clin Invest 129: 169-181, 2019.

35. Roos AB, Sanden C, Mori M, Bjermer L, Stampfli MR and Erjefalt JS: IL-17A is elevated in end-stage chronic obstructive pulmonary disease and contributes to cigarette smoke-induced lymphoid neogenesis. Am J Respir Crit Care Med 191: 1232-1241, 2015.

36. Zheng X, Zhang L, Chen J, Gu Y, Xu J and Ouyang Y: Dendritic cells and Th17/Treg ratio play critical roles in pathogenic process of chronic obstructive pulmonary disease. Biomed Pharmacother 108: $1141-1151,2018$

37. Ito JT, Cervilha DAB, Lourenco JD, Goncalves NG, Volpini RA, Caldini EG, Landman G, Lin CJ, Velosa APP, Teodoro WPR, et al: Th17/Treg imbalance in COPD progression: A temporal analysis using a CS-induced model. PLoS One 14: e0209351, 2019.

38. Li XN, Pan X and Qiu D: Imbalances of Th17 and Treg cells and their respective cytokines in COPD patients by disease stage. Int J Clin Exp Med 7: 5324-5329, 2014.

This work is licensed under a Creative Commons Attribution-NonCommercial-NoDerivatives 4.0 International (CC BY-NC-ND 4.0) License. 